\title{
Isolated fallopian tube torsion: a rare diagnosis in a perimenopausal patient with adnexal mass and raised Ca 19-9
}

\section{Endoscopic management and review}

Received: 12 April 2005/ Accepted: 29 July 2005/Published online: 9 September 2005

(C) Springer-Verlag Berlin / Heidelberg 2005

\begin{abstract}
Isolated torsion of the fallopian tube in perimenopausal women is very rare. A patient who presented with an adnexal mass with possible ovarian torsion and with a raised $\mathrm{Ca} 19-9$ titer is reported. A 48year-old perimenopausal woman had acute lower right abdominal pain accompanied by nausea and vomiting. Ultrasonographic studies revealed a multiloculated cystic mass in the right adnexum, and Ca 19-9 was raised. The symptoms worsened, and emergency laparoscopy and salpingoophorectomy were performed. Histology revealed isolated fallopian tube torsion with a benign ovarian cyst. Torsion of the fallopian tube, although rare, should always be considered as part of the differential diagnosis of any symptomatic pelvic mass, even in a perimenopausal woman. Characteristic ultrasonographic and CT findings have been reported, an early diagnosis can be made, and an endoscopic, less radical procedure will be the treatment of choice resulting in shorter hospitalization, increased patient satisfaction, and more cost benefits.
\end{abstract}

\section{Introduction}

Torsion of the normal or cystic ovary is a well-known clinical entity that usually involves the fallopian tube and ancillary structures. Isolated torsion of the fallopian

\footnotetext{
A. Daponte $(\bowtie) \cdot K$. D. Dimopoulos $\cdot$ I. E. Messinis Department of Obstetrics and Gynecology,

University of Thessalia, University Hospital of Larissa, 41110 Larissa, Greece

E-mail: dapontea@otenet.gr

Tel.: + 30-2410-672505

Fax: + 30-2410-672506

\section{A. Terzis}

Department of Pathology and Pathologic Anatomy, University of Thessalia, University Hospital of Larissa, 41110 Larissa, Greece
}

tube is, however, a rare condition, which was first described by Bland-Sutton in 1890 [1].

It is estimated to have an incidence of about 1 in 1.5 million women and involves particularly adolescents and young adults [2]. In the literature there have been 400 reported cases of which only one recently has referred to a perimenopausal woman [3].

To our knowledge, there have been no reports of isolated tubal torsion of a perimenopausal female patient with a raised $\mathrm{Ca} 19-9$, as in our case.

\section{Case report}

A 48-year-old gravida 3, para 1, perimenopausal woman on the 11th day of her cycle presented to our department complaining of the acute onset of severe right lower quadrant pain, which had started about 10-12 h earlier, radiating to the anterior aspect of the right thigh and was associated with nausea and vomiting. The pain had been localized since its onset and was colicky in nature, and there was no history of similar episodes. Bowel sounds were present and her bowel function during the previous week was reported to be normal. Her medical history included appendectomy, arterial hypertension, and irritable bowel. There was no family history of cancer. On physical examination her temperature and vital signs were within normal limits. Abdominal examination revealed tenderness and guarding in the right iliac fossa with no rebound tenderness. Examination with a speculum revealed no obvious infection. Swabs and a Pap smear were taken. On bimanual examination she had severe cervical motion tenderness and right adnexal tenderness with a mass of about $7 \mathrm{~cm}$ in size.

Urine pregnancy test and urine analysis were negative. Her white blood cell count was $4,800 / \mathrm{ml}$, hemoglobin was $14 \mathrm{~g} / \mathrm{dl}$, and serum $\mathrm{Ca} 125$, Ca 15-3, aFP, CEA, and $\beta$ HCG were within normal limits. There was a marked increase in the serum $\mathrm{Ca}$ 19-9 value $(109.2 \mu / \mathrm{ml}$; normal range $0-37 \mu / \mathrm{ml})$. The electrochem- 
iluminescence immunoassay (ECLIA) was used (monoclonal antibody 1116-NS-19-9) for measurement.

Transvaginal ultrasound scan showed a multiloculated cystic mass in the right adnexum, consisting of a cystic structure approximately $40 \times 50 \mathrm{~mm}$ in diameter on the right ovary and a multiloculated mass next to it compatible with hydrosalpinx or tubo-ovarian abscess (Figs. 1, 2). In the left ovary a follicle measuring $20 \times 20 \mathrm{~mm}$ in diameter was found. Doppler studies of both ovaries showed normal blood flow, while an abdominal X-ray was normal. Conservative treatment with antibiotics, analgesia, and nonsteroid anti-inflammatories was instituted. Prophylactic anticoagulation with low molecular weight heparin was given. The pain and vomiting subsided and her condition improved. She was booked for a CT scan and the possibility of explorative laparotomy and frozen section was discussed with her. She was very hesitant to follow that option.

On the 3rd day of her hospitalization, in the middle of the night, her condition started to deteriorate with exacerbation of the pain. She was advised that laparoscopy at least was needed and an adnexectomy was very likely, even if untwisting the potential torsion was possible since she had an ovarian cyst and raised Ca 19-9. She agreed to have a second operation, should the final pathology reveal malignancy or borderline tumor and insisted on not having a more extensive operation during the initial laparoscopy. Therefore, frozen section was omitted. A laparoscopic adnexectomy, with the presumptive diagnosis of intermittent ovarian torsion, was planned and the patient was taken to the operating room.

Laparoscopy revealed that the right ovary had a cystic mass about $4 \mathrm{~cm}$ in diameter, but was not twisted, that the left ovary had a follicle, and that the right fallopian tube was dark purple in color, was distended, and twisted on its vascular pedicle. The left fallopian tube and the uterus were normal and there were no ascites present. The right fallopian tube could not be untwisted. Despite repeated efforts it persisted in turning back to its original twisted position and the distal part remained engorged and dark. It was decided to limit the

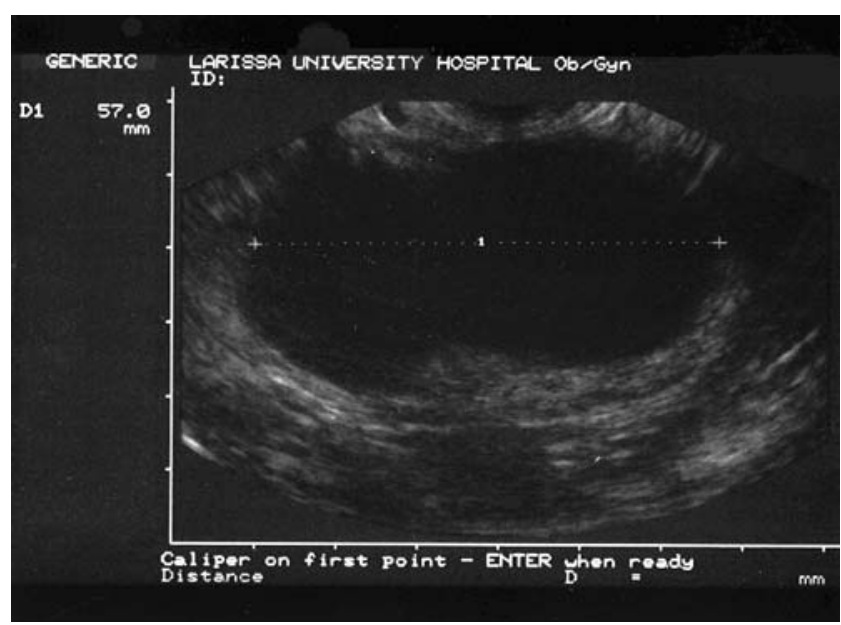

Fig. 1 The multiloculated cystic mass in the right adnexa

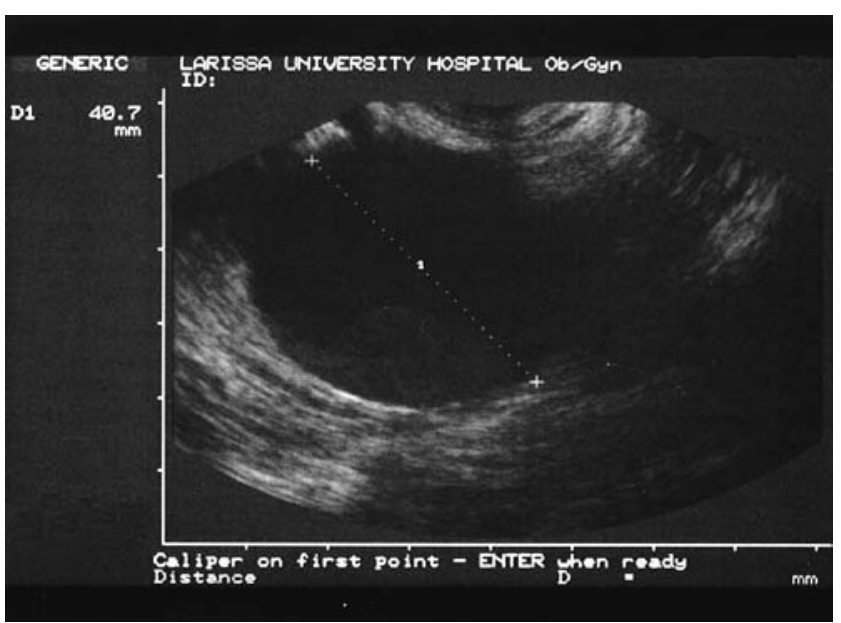

Fig. 2 The cystic structure on the right ovary

manipulations since we did not want to risk a rupture of the cyst. The tube was deemed not viable and considering the perimenopausal status of the patient after obtaining a peritoneal washing specimen, a right salpingoophorectomy was performed. Bipolar diathermy and an Endobag were used in order to prevent spillage of the cyst content. Cytology of the peritoneal washing specimen was negative.

The pathology report confirmed a $100 \times 18-\mathrm{mm}$ hemorrhagic, necrotic fallopian tube with extensive infarction secondary to torsion (Fig. 3). There were no features of a congenital abnormality of the fallopian tube. The right ovary was essentially normal with a corpus luteal cyst.

The diagnosis of isolated torsion of the fallopian tube was made. The postoperative course was uneventful and the patient was discharged 2 days later. She was very pleased that she had avoided the laparotomy and that her uterus and ovary had been preserved.

\section{Discussion}

The diagnosis of isolated fallopian tube torsion is often not a clinical one, but, as in our case, is made during

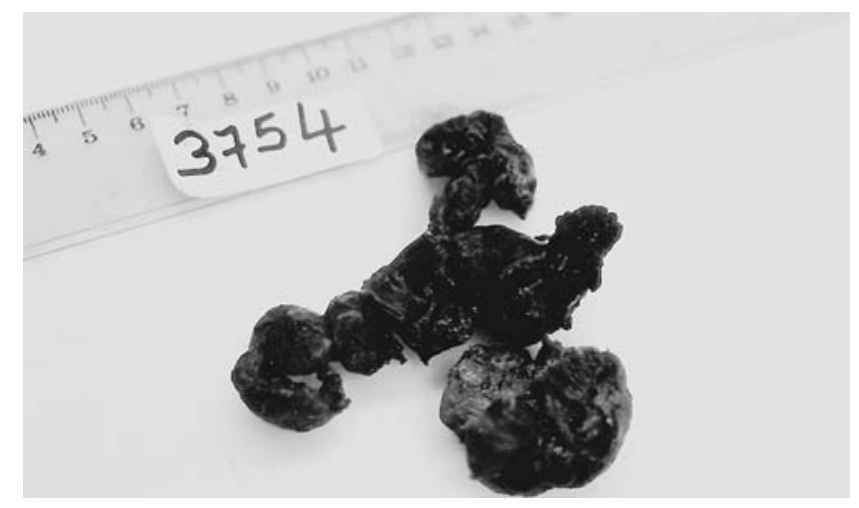

Fig. 3 The hemorrhagic, necrotic fallopian tube with the right ovary 
laparotomy or laparoscopy performed for the investigation or management of acute lower abdominal pain [4]. The differential diagnoses include ectopic pregnancy, ovarian torsion, acute appendicitis, ruptured ovarian cyst, pelvic inflammatory disease, and renal colic [5] .Acute appendicitis and ovarian torsion are the two most frequent misdiagnoses associated with fallopian tube torsion $[4,6]$. Torsion of the right fallopian tube, as in our case, is almost twice as common as the left in the literature by a $3: 2$ to $2: 1$ ratio, possibly because of the cushioning effect of the sigmoid colon on the left as well as a greater tendency toward surgical exploration in patients with right-sided pain to exclude appendicitis $[6,7]$.

The etiology of tubal torsion is still uncertain, especially when this is not associated with torsion of the ovary. Extrinsic and intrinsic factors have been associated with isolated tubal torsion [8]. The presence of the 4-cm benign ovarian cyst was the only possible extrinsic factor in our case. Other extrinsic factors, like uterine enlargement, mechanical factors (movement of the neighboring hollow viscera, sudden movement) or pelvic congestion, were not present. The pathology report revealed no congenital tubal abnormality or any other acquired tubal pathology, which were the main intrinsic factors that had been reported before [8]

Symptomatology, although not pathognomonic, includes sudden onset of intermittent pain in either of the lower abdominal quadrants, often accompanied by nausea and vomiting as in our case. In contrast, in acute appendicitis, vomiting develops several hours after the onset of abdominal pain, which characteristically shifts from the midline to the right lower quadrant. Bimanual pelvic examination may reveal a tender adnexal mass, associated with tenderness on cervical manipulation, as in our patient [9]. White cell count is often, but not always, normal.

Conventional ultrasonography in isolated tubal torsion might show the thickened walls of a distended fallopian tube with thickened fimbriae and the presence of internal debris within the tube, as in our case (Fig. 1)[10].

Color Doppler ultrasonography may detect unilateral high impedance flow with reversed diastolic flow or absence of blood flow to the twisted adnexal, although the presence of normal flow does not always, as in our case, rule out torsion $[10,11]$.

There has been little documentation of the CT findings in adnexal torsion. Ghossain et al. reported a series of 10 patients with surgically proven torsion in which tubal thickening (defined as tubal diameter $>15 \mathrm{~mm}$ ) could be detected retrospectively in 8 , and tubal hemorrhage (defined as density $>50 \mathrm{HU}$ on unenhanced examinations) in 6 patients [12].

Further characteristic features of adnexal torsion on CT include a thickened broad ligament associated with inflammatory changes [3, 13] and a cystic mass of tubular configuration with tubular thickening and free peritoneal fluid [10] has also been reported.
Available procedures include either resection when the tube cannot be salvaged, or untwisting and fixation of the tube if torsion is incomplete and/or recent and the tubal tissues remain viable, primarily in young women wishing to preserve their fertility [7, 12]. The obvious effects on the fertility of a patient with fallopian tube torsion should be viewed under the possibility of subsequent torsion and necrosis of the contralateral adnexum, especially if the patient is young. Pregnancy has been reported after detorsion [14]. The differentiation between necrosis with strangulation of the twisted tube and potential viability is, nevertheless, extremely challenging, and there is the theoretical risk of pulmonary embolism if untwisting is performed after thrombosis of the vascular supply of the tube [12]. Kruger and Heller [15] found that the surgeon's description of the adnexae at the time of surgery was a reliable predictor of the microscopic appearance of the necrosis.

In our case a raised $\mathrm{Ca}$ 19-9 titer was observed and was in line with the results of Engelen et al. [16], those of Saks and Deckardt [3], and those of Harada et al. [17], reporting raised levels of $\mathrm{Ca}$ 19-9 in borderline ovarian tumors, in dermoid cysts, and in endometriosis respectively. Due to the perimenopausal status of our patient, we opted for extensive counseling regarding repeat staging laparotomy after histology was available and oophorectomy instead of extirpation of the cystic mass.

In conclusion, torsion of the fallopian tube, although rare, should always be considered in the differential diagnosis of acute lower abdominal pain or any symptomatic pelvic mass in a young as well as in a perimenopausal woman. Failure to make the correct diagnosis can lead to necrosis, irreversible damage to the fallopian tube, and even damage to the ovary. Preserving the tube was not an issue in our perimenopausal patient and she is the first reported case with a raised $\mathrm{Ca}$ 19-9, although this may be an accidental finding. Explorative laparotomy, ideally by a gynecological oncologist, would be the standard management in a perimenopausal woman with an adnexal mass and raised tumor markers. This could be replaced by laparoscopic adnexectomy with peritoneal washings and frozen section, when the diagnosis is suspected preoperatively due to sonographic or CT features, as reviewed, and when ovarian pathology coexists in a perimenopausal patient, as in our case. After this rare entity has been included in the differential diagnosis, sonography, CT, and color Doppler will allow an early diagnosis (Table 1), and individualized surgical management. Laparoscopic detorsion, with preservation of the fallopian tube is the operation of choice, especially in young women wishing to preserve their uterus and other adnexum, or if it fails as in our case, laparoscopic salpingectomy. The patient must have extensive counseling regarding additional treatment should the final pathology not be benign, and peritoneal washings and endoscopic bag should be used. An early diagnosis and an endoscopic procedure will allow patient satisfaction and a shorter hospitalization, with obvious cost benefits. 
Table 1 Sonographic and CT features in the differential diagnosis of fallopian tube torsion

\section{Features}

Ultrasound/Doppler

Thickened walls of a distended fallopian tube with thickened fimbriae and the presence of internal debris within the tube [10]

Unilateral high impedance flow with reversed diastolic flow or absence of blood flow to the twisted adnexal $[10,11]$ CT

Tubal thickening defined as tubal diameter $>15 \mathrm{~mm}$ [12]

Tubal hemorrhage defined as density $>50 \mathrm{HU}$ on unenhanced examinations [12]

Thickened broad ligament associated with inflammatory changes [13]

Cystic mass of tubular configuration with tubular thickening and free peritoneal fluid [10]

\section{References}

1. Bland-Sutton JB (1890) Salpingitis and some of its effects. Lancet 2:1146

2. Hansen OH (1970) Isolated torsion of the fallopian tube. Acta Obstet Gynecol Scand 49:3-6

3. Saks M, Deckardt R (1994) Laparoscopic treatment of benign ovarian dermoid cysts. J Am Assoc Gynecol Laparosc 1:S31S32

4. Nichols DA, Julian PJ (1985) Torsion of the adnexa. Clin Obstet Gynecol 28:375

5. Wheeler JE (1985) Diseases of the fallopian tube. In: Kurman RJ (ed) Blaustein's pathology of the female genital tract. Springer, Berlin Heidelberg New York, pp 409-413
6. Provost RW (1972) Torsion of the normal fallopian tube. Obstet Gynecol 29:80

7. Filtenborg TA, Hertz JB (1981) Torsion of the fallopian tube. Eur J Obstet Gynecol Reprod Biol 12:177

8. Youssef AF, Fayad MM, Shafeek MA (1962) Torsion of the fallopian tube. Acta Obstet Gynecol Scand 41:292-309

9. Krissi H, Orvieto R, Dicker D, Dekel A, Rafael ZB (1997) Torsion of a fallopian tube following Pomeroy tubal ligation: a rare case report and review of the literature. Eur $\mathbf{J}$ Obstet Gynecol Reprod Biol 72:107-109

10. Richard HM III, Parsons RB, Broadman KF, Shapiro RS, Yeh HC (1998) Torsion of the fallopian tube: progression of sonographic features. J Clin Ultrasound 26:374-376

11. Baumgartel PB, Fleischer AC, Cullinan JA (1996) Color Doppler sonography of tubal torsion. Ultrasound Obstet Gynecol 7:367

12. Ghossain MA, Buy JN, Bazot M, Haddad S, Guinet C, Malbec L, Hugol D, Truc JB, Poitout P, Vadrot D (1994) CT in adnexal torsion with emphasis on tubal findings: correlation with US. J Comput Assist Tomogr 18:619-625

13. Skinner S, Voyvodic F, Scroop R, Sanders T (2001) Isolated tubal torsion: CT features. Clin Radiol 56:155-165

14. Blair CB (1962) Torsion of the fallopian tube. Surg Gynecol Obstet 114:727

15. Kruger E, Heller DS (1999) Adnexal torsion. A clinicopathologic review of 31 cases. J Reprod Med 44:71-75

16. Engelen MJ, de Bruijn HW, Hollema H, ten Hoor KA, Willemse PH, Aalders JG, van der Zee AG (2000) Serum CA 125, carcinoembryonic antigen, and CA $19-9$ as tumor markers in borderline ovarian tumors. Gynecol Oncol 78:16-20

17. Harada T, Kubota T, Aso T (2002) Usefulness of CA19-9 versus CA125 for the diagnosis of endometriosis. Fertil Steril 78:733-739 